\title{
Influence of Machining Parameters on Fractal Characterization of Typical Metallic Surface Topography
}

\author{
Wei Jiang ${ }^{1, a}$, Cuicui $\mathrm{Ji}^{2, \mathrm{~b}}$, Huiying Cao ${ }^{1, \mathrm{c}}$ \\ ${ }^{1}$ School of Mechanical and Automobile Engineering, Changzhou Institute of Technology, \\ Changzhou, Jiangsu 213032, PR China \\ ${ }^{2}$ School of Mechanical and Electrical Engineering, Hohai University, \\ Changzhou, Jiangsu 213022, PR China

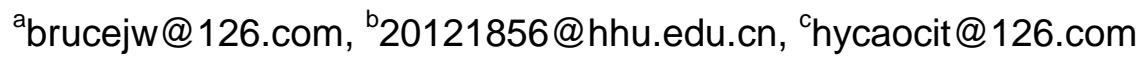

Keywords: machining parameters; fractal characterization; surface topography

Abstract. Based on the fractal theory, this paper quantitatively studies the influence of machining parameters on fractal characterization of typical metallic surface topography. Seven kinds of materials were selected including Q235, 45\#, 0Cr18Ni9, HT200, T2, HPb59-1 and YL12. The surface topography was obtained under different machining parameters and the corresponding fractal dimension $D$ and characteristic roughness $R a^{*}$ were calculated using root mean square method. The conventional parameter roughness $R a$ was used for comparison to evaluate the influence of turning speed and feed rate and tries to reveal the feasibility of fractal parameters to characterize the machinability of materials and eventually establish the relationship between fractal parameters and material properties.

\section{Introduction}

Since the foundation of fractal geometry by Mandelbort in 1982 [1], the research of fractal characteristics of surface topography has attracted wide attention and the involvement of fractal theory provides a new approach to characterize complicated surface topography quantitatively. The fractal theory has been widely used and proved to be a useful tool for the research of micro structure, contact mechanism and surface topography of machined surface [2-5]. The research shows that the machined surface is random, multi-scale and self-affine, which has the basic feature of fractal. As a result, fractal geometry can be used for the analysis of surface topography effectively.

As one of the most important parameters of fractal geometry, fractal dimension $D$ expresses the complexity of the surface structure. The larger the fractal dimension $D$, the more irregular structure exists in the surface topography, which is superior to the traditional evaluation parameters for characterization of the degree of surface roughness and can provide more information than traditional surface roughness evaluation parameters. In recent years, with the further research of fractal theory, there are various methodologies for calculating the fractal dimension were proposed such as structure function method and root mean square method. The root mean square method was validated to be a more suitable approach for the characterization of the rough surface and a new fractal characterization parameter was proposed by $\mathrm{Ge}$ and $\mathrm{Zhu}$ as characteristics roughness $R a^{*}$ [6,7]. In this paper, seven kinds of typical metallic materials were machined using turning machine under different machining parameters to obtain corresponding surface topography. Fractal parameters such as scale coefficient $C$, fractal dimension $D$ and characteristic roughness $R a^{*}$ were introduced to evaluate the influence of machining parameters on the fractal characterization of surface topography with combination of traditional characterization parameter $R a$. The feasibility of fractal parameters to characterize the machinability of materials was analyzed and the relationship between fractal parameters and material properties could be eventually established. 


\section{Materials and Methods}

With consideration of various factors, seven kinds of typical metal materials were used including Q235, 45\#, 0Cr18Ni9, HT200, T2, HPb59-1 and YL12. The corresponding surface topography was obtained using turning method under different machining parameters including turning speed and feed rate, which is demonstrated in Table 1.

During the machining process, the YT15 tool was used and the shape of the tool was ensured to be stable. Meanwhile, the cutting depth remains $0.1 \mathrm{~mm}$ and the turning speed range from 37 to $820 \mathrm{rpm}$ and the corresponding feed rate range from 0.08 to $0.28 \mathrm{~mm} / \mathrm{r}$. T1000 profilometer was used to carry out multiple measurements on the surface topography to obtain digital data for fractal analysis with sampling points 4096 and sampling length $2.5 \mathrm{~mm}$. Afterwards, the root mean square method was applied to calculate the scale coefficient $C$, fractal dimension $D$ and characteristic roughness $R a^{*}$ using MATLAB to observe the influence of machining parameters on the fractal characterization. The surface topography obtained under the same processing parameters were used to analyze the influence of material properties on fractal characterization.

Table 1 Machining parameters

\begin{tabular}{ccc}
\hline Tool Material & \multicolumn{2}{c}{ Specimen Material(Ф30mm) } \\
\hline YT15 & Q235, 45\#, 0Cr18Ni9, HT200, T2, HPb59-1, YL12 \\
\hline Turning Speed(r/min) & Feed Rate $(\mathrm{mm} / \mathrm{r})$ & Depth(mm) \\
\hline $37,150,410,820$ & $0.08,0.14,0.20,0.28$ & 0.1 \\
\hline
\end{tabular}

\section{Fractal Characterization}

Fractal Dimension $\boldsymbol{D}$ In this paper, the fractal dimension $D$ of the surface topography was calculated using the root mean square method [6]. The power law relationship between root mean square measure $\sigma(\tau)$ and the scale $\tau$ is illustrated in Eq.1 [8].

$$
\sigma(\tau)=\left([Z(x)]^{2}\right)^{1 / 2}=\left[\frac{G^{2(D-1)}}{2 \ln \gamma} \frac{1}{4-2 D}\right]^{1 / 2} \tau^{2-D}=C \tau^{2-D}
$$

where $D$ is the fractal dimension; $C$ is the scale coefficient; $Z(x)$ is the W-M fractal function, its mathematical expression is demonstrated in Eq.2 [9].

$$
Z(x)=G^{(D-1)} \sum_{n=n_{l}}^{\infty} \gamma^{-(2-D) n} \cos \left(2 \pi \gamma^{n} x\right)
$$

With a logarithm operation to the Eq.1, a straight line equation can be obtained in Eq.3.

$$
\lg \sigma(\tau)=\lg C+(2-D) \lg \tau
$$

Thus the fractal dimension $D$ can be calculated from the slope of the line and the scale coefficient $C$ from the linear intercept.

Characteristic Roughness $\boldsymbol{R} \boldsymbol{a} *$ Due to the fact that neither fractal dimension $D$ nor scale coefficient $C$ can exclusively determine a surface. Therefore, the characteristic roughness parameter $R a^{*}$ was presented to characterize surface topography which combines the fractal dimension $D$ and scale coefficient $C$ and its mathematical expression is illustrated in Eq. 4 [7]. 


$$
R a^{*}=C^{-1(2-D)}
$$

With the combination of fractal dimension $D$ and scale coefficient $\mathrm{C}$, characteristic roughness $R a^{*}$ can not only reflect the complexity of surface topography which fractal dimension $D$ reflects, but also can reflect the roughness level which scale coefficient $C$ meant to reflect. Therefore, characteristic roughness $R a^{*}$ can characterize the surface topography comprehensively.

\section{Results and Discussion}

Figs. 1-3 demonstrate the influence of machining parameters such as turning speed and feed rate on the fractal characterization of seven kinds of typical metal materials, including surface roughness $R a$, fractal dimension $D$ and characteristics roughness $R a^{*}$ respectively. Due to limited space, only part of the data was elaborated in this paper. The feed rate was $0.2 \mathrm{~mm} / \mathrm{r}$ when analyzing the influence of the turning speed on fractal characterization and the turning speed was set as $410 \mathrm{rpm}$ when analyzing the influence of feed rate. It should be cleared that surface roughness $R a$, fractal dimension $D$ and characteristics roughness $R a^{*}$ have the same variation under other machining parameter.
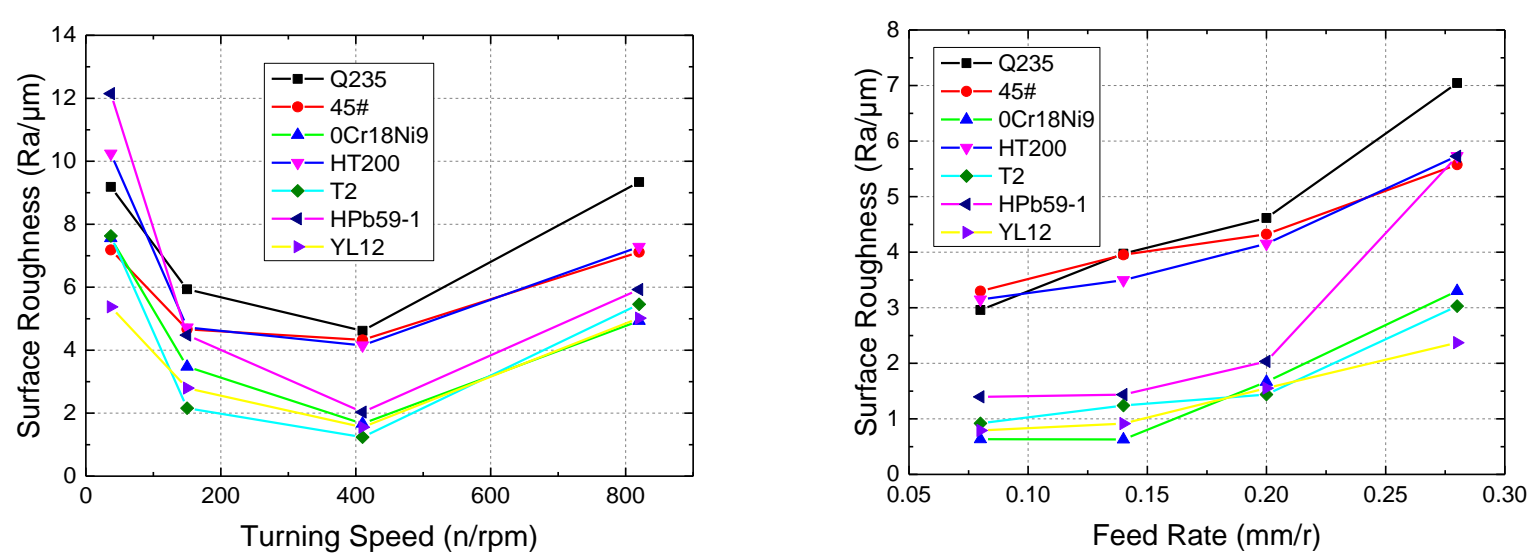

Figure 1: Influence of machining parameters on surface roughness $R a$.

As can be seen from Fig.1, when the feed rate remains constant, the surface roughness $R a$ show a trend of decreasing first and then increasing with the increasing of turning speed. This is because plastic deformation of materials reduces with the increasing of turning speed and the surface roughness $R a$ reaches the lowest value at 410rpm. However, the vibration of processing system increases when the turning speed increased continuously and the resultant surface quality becomes poor. In another aspect, when the turning speed is kept constant, the surface roughness $R a$ increases with the increase of the feed rate, which is due to the residual area height of the workpiece surface and the contact length between tool and chip increases. Therefore, the production probability of built-up edge and the plastic deformation increases, which result in larger value of surface roughness $R a$.

As illustrated in Fig.2 and Fig.3, the variation of fractal dimension $D$ is similar to characteristic roughness $R a^{*}$. That is, when feed rate remains constant, both fractal dimension $D$ and characteristic roughness $R a^{*}$ increase first and then decrease with the increasing of turning speed, and have a maximum value as the turning speed raised to $410 \mathrm{rpm}$. When the turning speed is constant, both fractal dimension $D$ and characteristic roughness $R a^{*}$ show a decreasing trend with the increasing of feed rate. It is worth noting that the feed rate has more significant influence on both fractal dimension $D$ and characteristic roughness $R a^{*}$ than that of turning speed. As the characteristic roughness $R a^{*}$ has the effect of superposition and amplification of the fractal dimension $D$ and scale coefficient $\mathrm{C}$, the characterization sensitivity of characteristic roughness $R a^{*}$ is much higher than that of fractal dimension $D$. 

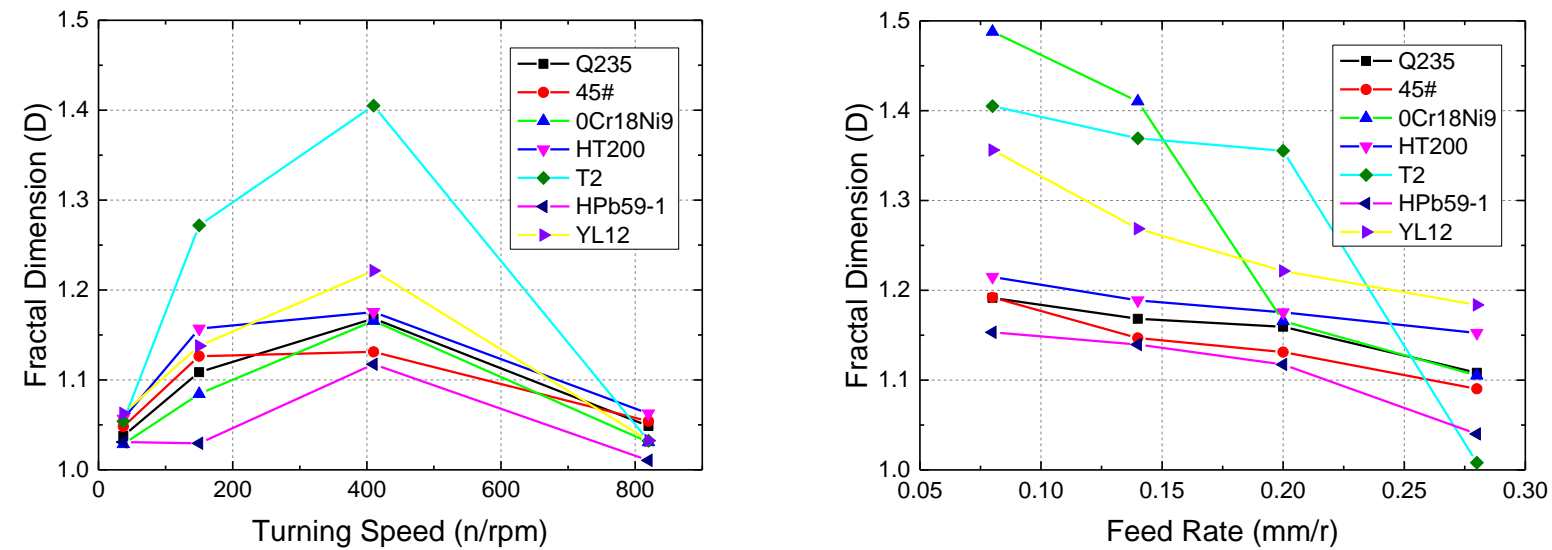

Figure 2: Influence of machining parameters on fractal dimension $D$.
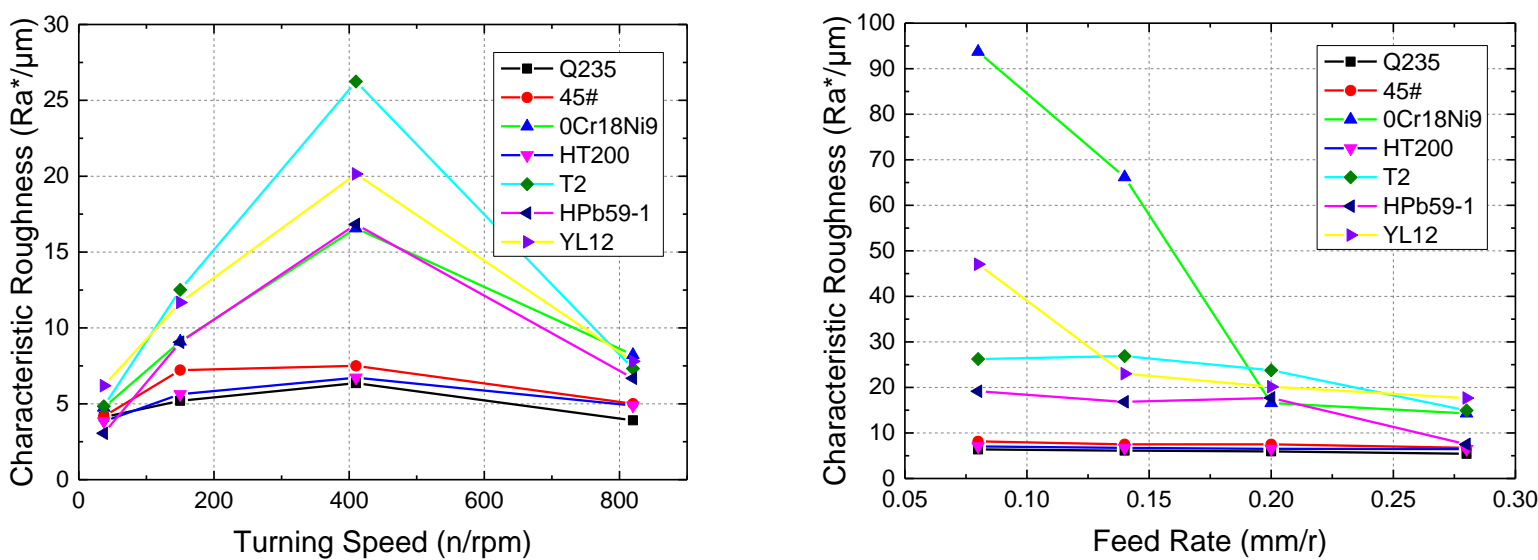

Figure 3: Influence of machining parameters on characteristics roughness $R a^{*}$.
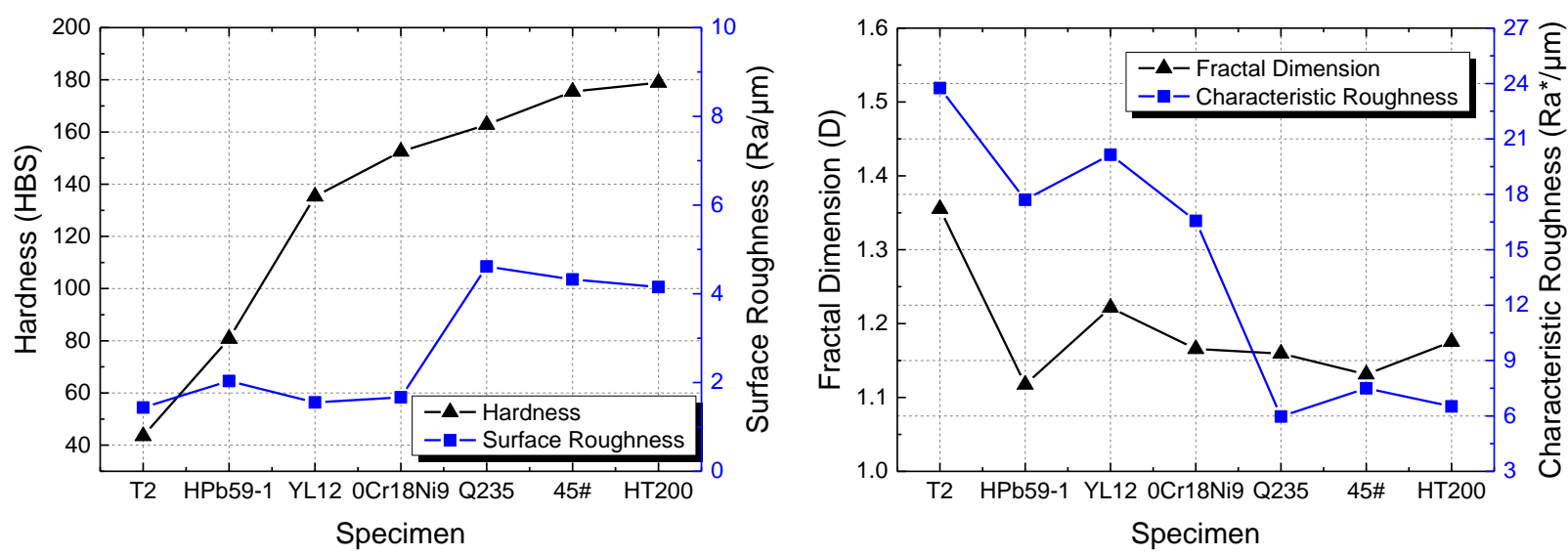

Figure 4: Influence of material properties on fractal characterization.

In order to study the influence of material properties on the fractal characterization, the surface topography obtained when turning speed and feed rate was set as $410 \mathrm{rpm}$ and $0.2 \mathrm{~mm} / \mathrm{r}$ respectively was used as research object. The fractal parameters vary with different workpiece material properties under the same machining conditions. In general, the higher hardness of the workpiece, the more difficult for machining. The Brinell Hardness of seven kinds of metal material was measured using sclerometer. As illustrated in Fig. 4, with the increase of the hardness, the surface roughness $R a$ increases. In contrast, the fractal dimension $D$ and the characteristic roughness $R a^{*}$ show a downward trend, however, no obvious relationship found. It is worth noting that although the surface roughness $R a$ of T2, HPb59-1, YL12 and 0Cr18Ni9 is relatively close, the fractal dimension $D$ and the 
characteristic roughness $R a^{*}$ are quite different. The surface roughness $R a$ of Q235, 45\# and HT200 is close and higher than other metal materials and there is no major difference in fractal dimension $D$ and characteristic roughness $R a^{*}$. From the point of view of the machinability, the value of fractal dimension $D$ and characteristic roughness $R a^{*}$ may be able to characterize the machinability of materials. However, this work needs further research to reveal the feasibility and finally establish the relationship between fractal parameters and material properties.

\section{Conclusions}

Based on the presented analysis and discussion, the following main conclusions can be drawn from this study.

(1) The fractal dimension $D$ and characteristic roughness $R a^{*}$ increases first and then decreases with the increasing of turning speed and both decreases with the increase of feed rate, and the feed rate has greater influence than that of turning speed.

(2) Characteristic roughness $R a^{*}$ has much higher characterization sensitivity than that of fractal dimension $D$ due to its superposition and amplification effect of fractal dimension $D$ and scale coefficient $C$.

(3) Fractal dimension $D$ and characteristic roughness $R a^{*}$ decreases with the increasing of hardness of workpiece under the same machining conditions, fractal parameters may be able to characterize the machinability of materials, which need to be further studied.

\section{Acknowledgements}

This work was financially supported by the Applied Basic Research Programs of Science and Technology Commission Foundation of Jiangsu Province (BK20150256), Scientific Research Foundation of Changzhou Institute of Technology (YN1512) and CIT Training Program of Innovation and Entrepreneurship for Undergraduates (J150186).

\section{References}

[1] B. B. Mandelbrot. The Fractal Geometry of Nature[M]. New York: W. H Freeman and Co. 1982.

[2] L. He, J. Zhu. The Fractal Character of Processed Metal Surfaces[J]. Wear, 1997, 208: 17-24.

[3] P. Sahoo, T. Barman, J.P. Davim. Fractal Analysis in Machining[J]. Springerbriefs in Applied Sciences \& Technology, 2011, 3: 69-81.

[4] C.C. Ji, H. Zhu, W. Jiang, Running-in Test and Fractal Methodology For Worn Surface Topography Characterization[J]. Chinese Journal of Mechanical Engineering, 2010, 23(5): 600-605.

[5] D.S. Li, Y.D. Zhang, Q. Wu, P. Wang. Identification of Fractal Scale Parameter of Machined Surface Profile[J]. Applied Mechanics and Materials, 2011, 42: 209-214.

[6] H. Zhu, S.R. Ge. Comparison of Fractal Characterization Effects of Structure Function and Mean Wquare Root[J]. Journal of China University of Mining \& Technology, 2004, 33(4): 396-399.

[7] H. Zhu, S.R. G, X.L. Huang, et al. Experimental Study on the Characterization of Worn Surface Topography with Characteristic Roughness Parameter[J]. Wear, 2003, 255: 309-314.

[8] S.R. Ge, K. Tonder. The Fractal Behavior and Fractal Characterization of Rough Surfaces[J]. Tribology, 1997, 17(1): 73-80.

[9] M.V. Berry, Z.V. Lewis. On the Weierstrass-Mandelbrot Fractal Function[J]. Proc. R. Soc. London, Ser. A, 1980, 370: 459-484. 